\title{
Herramientas de trabajo colaborativo para la dinamización de la competencia transversal responsabilidad ética, medioambiental y profesional
}

\section{M.T. Sebastiá-Frasquet ${ }^{\mathrm{a}}$, S. Asensio-Cuesta ${ }^{\mathrm{b}}$, I. Gasch ${ }^{\mathrm{c}}$, N. Pascual-Seva ${ }^{\mathrm{d}}$, M. Vargas ${ }^{\mathrm{e}}$}

${ }^{a}$ Dpto de Ingeniería Hidráulica y Medio Ambiente. Universitat Politècnica de València (mtsebastia@hma.upv.es) b Dpto. de Proyectos de Ingeniería. Universitat Politècnica de València (sasensio@dpi.upv.es). ${ }^{\mathrm{D} D}$ pto. de Mecánica de los Medios Continuos y Teoría de Estructuras. Universitat Politècnica de València (igasch@mes.upv.es). ${ }^{\mathrm{d}}$ Dpto. de Producción Vegetal. Universitat Politècnica de València (nupasse@prv.upv.es). ${ }^{e}$ Dpto. de Tecnología de Alimentos. Universitat Politècnica de València (mavarco@tal.upv.es).

\begin{abstract}
This article analyzes the experience of using different collaborative work tools to dynamize the tasks that help to acquire and improve key competence ethical, environmental and professional responsibility. The selected tools are integrated in the learning management system (LMS), PoliformaT, of the Universitat Politècnica de València. In particular, we have worked with the forum and the wiki, and the blog which is integrated in Poliblogs. In this experience three Bachelor degrees and a Master degree of engineering and life sciences have been involved: Bachelor's Degree in Environmental Sciences, Bachelor's Degree in Rural and Agrifood Engineering, Bachelor's Degree in Mechanical Engineering and Master's Degree in Design Engineering. The results show that the students value the experience satisfactorily thanks to the virtual interaction with other colleagues and they highlight the extra motivation offered by the use of these tools. It is noteworthy that to obtain good results there are two key elements: 1) clear rules of operation and evaluation and 2) high participation of the teacher as moderator.
\end{abstract}

Keywords: blog, forum, wiki, learning management system, key competences

\section{Resumen}

En el presente artículo se analiza la experiencia de uso de distintas herramientas de trabajo colaborativo para dinamizar las tareas que ayudan a adquirir y mejorar la competencia transversal responsabilidad ética, medioambiental y profesional. Las herramientas seleccionadas están integradas en la plataforma de gestión del aprendizaje, poliformat, de la Universitat Politècnica de València. En particular hemos trabajado con el foro y la wiki, además de con el blog integrado en Poliblogs. En esta experiencia han participado tres titulaciones de Grado y un Máster de ingeniería y ciencias de la vida: Grado en Ciencias Ambientales, Grado en Ingeniería Agroalimentaria y del Medio Rural, Grado en Ingeniería Mecánica, y Máster Universitario en Ingeniería del Diseño. Los resultados obtenidos muestran que el alumnado valora satisfactoriamente la experiencia gracias a la interacción 
virtual con otros compañeros y destacan la motivación extra que les ofrece el uso de estas herramientas. Es destacable que para la obtención de buenos resultados hay dos elementos clave: 1) Normas claras de funcionamiento y evaluación y 2) Alta participación del profesor como moderador.

Palabras clave: blog, foro, wiki, sistemas de gestión del aprendizaje, competencias transversales

\section{Introducción}

Las competencias transversales son habilidades relacionadas con el desarrollo personal útiles en múltiples áreas (González et al., 2003). Las competencias transversales constituyen una parte fundamental del perfil profesional y formativo del alumno.

En los últimos años, la Universidad Politécnica de València (UPV) ha desarrollado una nueva política de acreditación de competencias transversales en los planes de estudios de sus alumnos (UPV, 2017). En la UPV se han definido un total de trece competencias entre las que se encuentra la correspondiente a "Responsabilidad ética, ambiental y profesional" objeto del presente trabajo. Además, se han definido las rúbricas para evaluar estas competencias (Fernández et al., 2017).

El equipo de innovación docente que presenta este artículo lleva trabajando varios años sobre la competencia transversal "Responsabilidad ética, medioambiental y profesional", lo que se ha plasmado en diversas publicaciones (Pascual-Seva et al., 2015, Sebastiá-Frasquet et al., 2016, Asensio et al., 2017). Durante el curso académico 2016-2017 el equipo ha llevado a cabo su innovación bajo el Proyecto de Innovación y Mejora Educativa "A10: Herramientas de trabajo colaborativo para la dinamización del competencia transversal responsabilidad ética, medioambiental y profesional".

\section{Objetivos}

El principal objetivo es explorar el uso de herramientas de trabajo colaborativo para evaluar la competencia transversal de responsabilidad ética, medioambiental y profesional.

\section{Desarrollo de la innovación}

La innovación se ha desarrollado en dos partes diferenciadas por la herramienta de trabajo colaborativo utilizada. Las herramientas utilizadas han sido el blog, la wiki y el foro de la plataforma de aprendizaje PoliformaT de la Universitat Politècnica de València (UPV). La actividad de innovación se ha realizado durante el cuatrimestre A del curso académico 2016-2017.

\subsection{Blog}

El blog se ha utilizado en las siguientes asignaturas:

1) Necesidades y Programación del Riego y la Fertilización. Esta asignatura pertenece al $4^{\circ}$ curso de la especialidad Hortofruticultura y Jardinería del Grado en Ingeniería 
Agroalimentaria y del Medio Rural. En el curso en el que se realiza la innovación hay un total de 40 alumnos matriculados en la asignatura.

2) Estructuras de Hormigón Armado. Esta asignatura pertenece al $4^{\circ}$ curso de la especialidad Diseño Estructural del Grado en Ingeniería Mecánica. En el curso en el que se realiza la innovación hay un total de 22 alumnos matriculados en la asignatura.

3) Ergonomía en el Diseño. Esta asignatura se imparte en el Máster Universitario en Ingeniería del Diseño.

Se utiliza el mismo blog para las 3 asignaturas implicadas. El blog está desarrollado en Wordpress y se integra en los Poliblog de la UPV. El blog se ha denominado "Responsabilidad ética, medioambiental y profesional en ingeniería y ciencias de la vida" y se puede acceder en la siguiente dirección: http://responsabilidad.blogs.upv.es. La finalidad del blog es que los alumnos interactúen aportando evidencias relacionadas con la competencia tranversal abordada y generando un debate crítico en torno a estas evidencias que permita evaluarlos. Las evidencias son de dos tipos. Por una parte, en las asignaturas de "Necesidades y Programación del Riego y la Fertilización" y "Estructuras de Hormigón Armado", las evidencias aportadas son noticias y artículos científicos de la materia de la asignatura que abordan la responsabilidad profesional, ética y/o medioambiental. Por otra parte, en la asignatura "Ergonomía en el Diseño", la evidencia aportada por los alumnos es su propio proyecto desarrollado en la asignatura. En Ergonomía del Diseño se trabaja con aprendizaje basado en proyectos. El uso del blog permite que los estudiantes tengan acceso al proyecto de sus compañeros y se establece un debate crítico acerca de los criterios éticos y medioambientales que se deben tener en cuenta en el diseño. La experiencia en esta asignatura ha sido descrita de forma más extensa en Asensio et al. (2017).

En el primer caso se pretende evaluar el nivel de competencia transversal adquirida por los alumnos, por lo que se califica con los niveles A-D, establecidos por la UPV.

Las aportaciones de noticias, información, etc. al blog se evalúan siguiendo los siguientes criterios:

A-La aportación, relacionada con la CT "Responsabilidad ética, medioambiental y profesional”, tiene carácter científico (revistas científicas).

B-La aportación, relacionada con la CT "Responsabilidad ética, medioambiental y profesional", tiene carácter divulgativo (noticia de prensa, webs especializadas, etc.).

C-La aportación no está relacionada con la CT "Responsabilidad ética, medioambiental y profesional" o es poco relevante.

D-La aportación carece de sentido.

El debate se inicia por el estudiante que aporta la evidencia (artículo). El profesor evalúa las contribuciones de los estudiantes al debate siguiendo los criterios:

Inicio del debate: 
A-El comentario es apropiado y presenta un buen planteamiento.

B-Aunque el comentario presenta una idea buena, podría mejorarse.

C-El comentario no está justificado.

D-Se aporta la noticia sin comentar.

Resto de comentarios, aportados por los compañeros:

A-El comentario es apropiado, está debidamente justificado y elaborado.

B-El comentario es apropiado, pero no está debidamente justificado y/o elaborado.

C-El comentario no aporta ninguna idea nueva (e.g. Estoy de acuerdo con los compañeros)

D-El comentario carece de sentido.

Para alcanzar la máxima calificación (A) se requiere: una aportación con calificación A + un inicio de debate con calificación $\mathrm{A}+$ dos comentarios con calificación A.

Las profesoras participaron en la moderación del foro, contribuyendo con observaciones sobre los comentarios. Además, en clase se dedicó un tiempo breve al final de una sesión para mostrar a los alumnos las evidencias y comentarios. Esto se hizo para tratar de implicar a alumnos que no habían participado ya que la actividad no tenía en ningún caso carácter obligatorio.

\subsection{Wiki y foro}

La wiki y el foro se han utilizado en la asignatura optativa Groundwater Management in the Coastal Zone, del $4^{\circ}$ curso del Grado en Ciencias Ambientales. En el curso en el que se realiza la innovación hay un total de 19 alumnos matriculados, de los cuales 7 son alumnos de intercambio del programa Erasmus.

En esta asignatura se plantearon dos tipos de actividades a los alumnos, ha realizar con distintas herramientas, la wiki y el foro. A continuación, se describen las 2 experiencias.

La wiki se utilizó para elaborar información detallada sobre uno de los "hot topics" de la asignatura. Cabe decir, que la asignatura se imparte en inglés y la wiki se elaboró en inglés. En este caso es la profesora la que selecciona un tema de complejidad suficiente para tratar los distintos aspectos de la competencia transversal. Durante el curso 2016/17 se seleccionó la problemática de la zona húmeda de las Tablas de Daimiel. Esta zona experimentó graves incendios de turba durante el año 2009 debido a la sobreexplotación de los acuíferos. En la actualidad este ecosistema se ha recuperado parcialmente gracias a distintos factores. En este caso de estudio los factores ambientales son evidentes, pero también la ética y la responsabilidad profesional tienen un gran peso debido a la gran conflicitividad social que surge alrededor del aprovechamiento del recurso hídrico. La profesora elabora el esquema de la wiki y asigna a cada grupo de alumnos (parejas o grupos de tres máximo) uno de los apartados para que lo desarrollen. En una segunda etapa, cada grupo debe revisar y evaluar de forma crítica los apartados elaborados por el resto de compañeros. Los alumnos son

(cc) EY-NC-ND 2017, Universitat Politècnica de València 
evaluados tanto por los contenidos que desarrollan como por la capacidad de detectar las carencias en los contenidos de los compañeros.

El foro se utilizó tras una salida de campo que en el curso 2016/17 se realizó a la Junta de Usuarios del Alto Vinalopó. Esta actividad pretende ver en la práctica todo lo aprendido en la asignatura sobre la gestión integral de recursos hídricos. Durante la visita los técnicos de la Junta de Usuarios realizan una exposición sobre su funcionamiento y se plantea un debate de distintos factores sociales, políticos y económicos que tienen una clara vertiente de responsabilidad ética, profesional y medioambiental. El foro en el PoliformaT de la asignatura se inaugura al terminar la visita. La profesora plantea diversas preguntas que los alumnos deben contestar y razonar. La escala de valoración es al igual que en la experiencia descrita para el blog cualitativa. El foro estuvo abierto únicamente durante una semana, y en este periodo la profesora ejerció de moderadora.

\section{Resultados}

En todas las experiencias descritas el alumnado se mostró dispuesto a participar de forma activa. Sin embargo, en términos cuantitativos los resultados de participación muestran una clara diferenciación entre las asignaturas en las que la actividad era obligatoria y aquellas en las que era voluntaria. Tanto en "Ergonomía en el diseño" como en "Groundwater management" la participación fue del $100 \%$ dada la obligatoriedad de la tarea. En cambio, en las asignaturas "Necesidades y Programación del Riego y la Fertilización" y "Estructuras de Hormigón Armado" el porcentaje de participación fue inferior al 30\% de los matriculados (15 y $28 \%$ respectivamente).

El bajo porcentaje de participación en las asignaturas en las que la actividad era optativa se atribuye en gran medida a las dificultades técnicas con el uso del blog. El administrador/administradores del blog deben dar de alta a todos los alumnos que vayan a participar, para ello los alumnos tienen que haber entrado al menos una vez en Poliblogs previamente. Una vez dados de alta, les llega un correo electrónico en el que tienen que aceptar las condiciones de uso. En las 3 asignaturas en las que se utilizó el blog esto generó bastantes problemas. Se tuvo dificultades para dar de alta a los alumnos y se tuvieron que presentar diversas incidencias al servicio técnico de PoliformaT a través de la aplicación Gragal. Aún así, en dos de las asignaturas no se pudo completar el proceso de alta del 100\% de los matriculados.

En las siguientes figuras se muestran algunas de las contribuciones de los alumnos al blog. 
Herramientas de trabajo colaborativo para la dinamización del competencia transversal responsabilidad ética, medioambiental y profesional

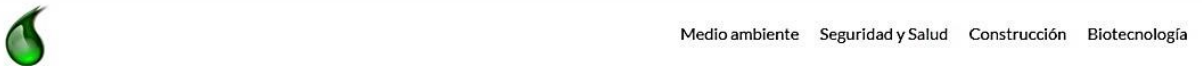

Desarrollan un hormigón compuesto por bacterias que se repara por sí solo

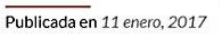

Investigadores holandeses han desarrollado un nuevo tipo de material de construcción que repara sus grietas sin ayuda externa, sino gracias a los microorganismos que hayensu interior.

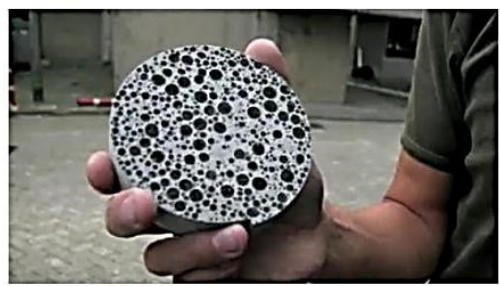

Este material constará de hormigón en sí, al que se le añadirán bacterias de origen biológico, dando lugar al llamado "biohormigonn. Este tiene la capacidad de repararse por si solo, al rellenar con la caliza sintetizada por los reforzado con acero que se puede oxidar por el contacto con el agua $y$ son estas bacterias las que impediran dich oxidación e incluso reconstruir las grietas por las que se filtra el agua. Este sistema aumenta la esperanza de vida de hormigón y permite ahorrar en reparaciones y mantenimiento de este.

A continuación, les dejo un enlace donde podrán obtener m información sobre este avance en la construcción:

COMENTARIOS RECIENTES

http://www.elconfidencial.com/tecnologia/2015-05-19/hormigon-cemento-bacterias-grietas-

juquisan en La agricultura ecológica valenciana busca ser "referente europeo"

Isabel Gasch Molina en Desarrollan un hormigón compuesto por bacterias que se repara por sísolo

rogiha en La paja del arroz. Consecuencias de su manejo y alternativas de aprovechamiento.

mirodpon en La agricultura ecológica valenciana busca ser "referente europeo"

samocna en Autorizado un sondeo de investigaciónpreexplotación contra la sequía en el río Serpis en Valencia 208 autorreparacion_807312/

Esta entrada fue publicada en Construcción. Guarda el permalink.

$\leftarrow$ Valdemoro construye su primer Edificio de Consumo de Energía Casi Nulo

ERGONOMÍA EN EL DISEÑO - SILLA DE PLAYA -

Fig. 1 Entrada al blog de un estudiante de la asignatura Estructuras de hormigón armado 


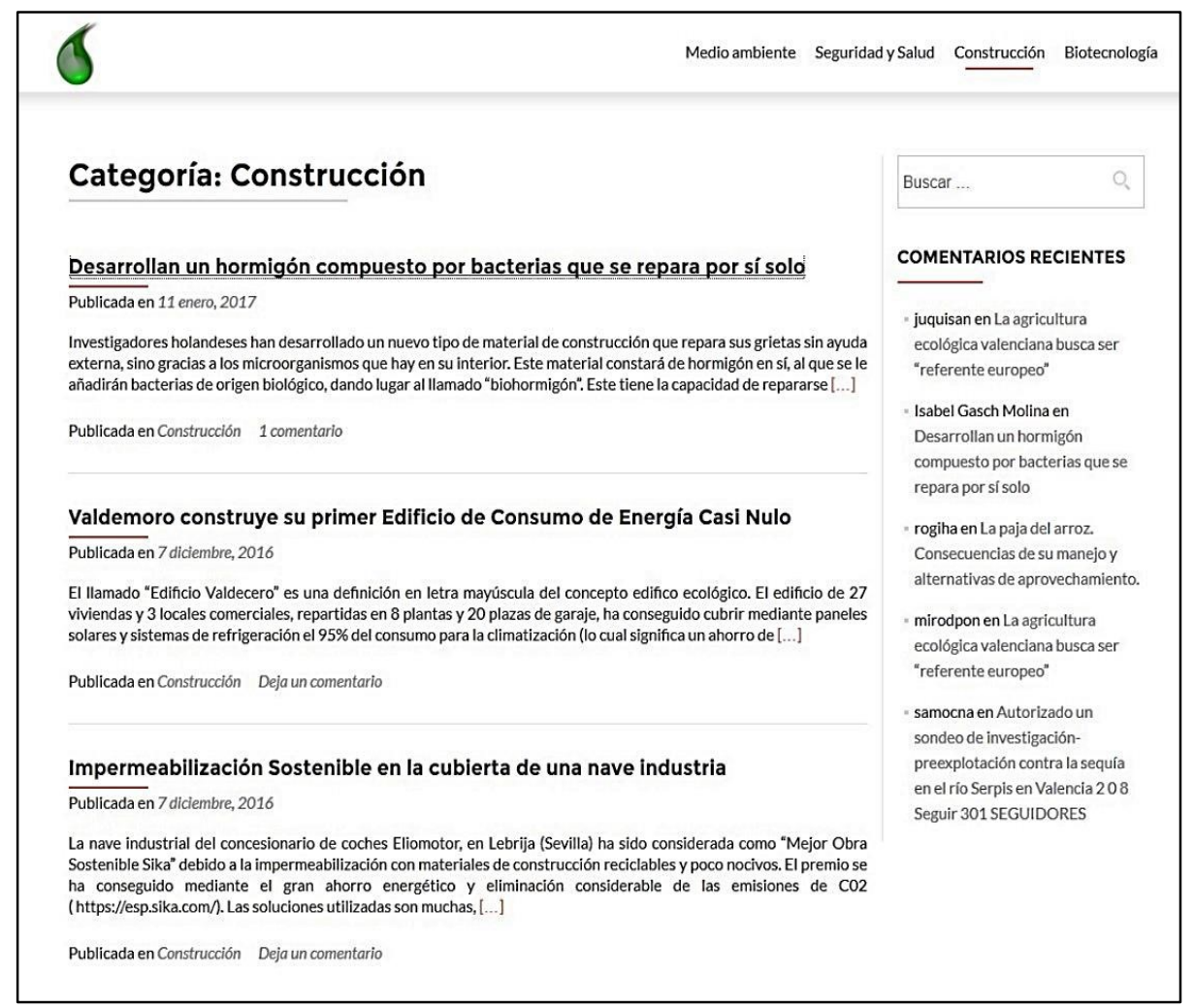

Fig. 2 Vista de varias entradas al blog de la asignatura Estructuras de hormigón armado

Las aportaciones de los alumnos a las asignaturas "Necesidades y Programación del Riego y la Fertilización" y "Estructuras de Hormigón Armado" fueron valoradas con una A, ya que fueron aportaciones de calidad, relacionadas con la competencia estudiada y los comentarios fueron significantes. El bajo porcentaje de participación pudo ser la causa de que todos los participantes estuvieran realmente motivados por participar en la actividad y de ahí el buen resultado obtenido.

En la asignatura de "Ergonomía en el diseño" los resultados observados fueron también buenos. Estos resultados están descritos en Asensio et al. (2017), pero cabe destacar que los alumnos valoraron el uso del blog de forma muy positiva. Ver su trabajo expuesto al público (profesora, compañeros de clase, expertos en la materia invitados y público en general) de forma "permanente" fue un factor motivador para tratar de hacer un buen trabajo. El diseño se evaluó en base a criterios ambientales y éticos. La responsabilidad profesional se demostró al tener que exponer su proyecto de forma profesional.

En la asignatura "Groundwater management in the coastal zone" se observaron los siguientes resultados. La creación de una wiki sobre el caso de estudio propuesto (Gestión del agua en las Tablas de Daimiel) fue valorada positivamente por los alumnos. La realización en 2 etapas de la actividad permitió a los alumnos analizar de forma crítica su propia contribución y la de sus compañeros. La nota media de la actividad de los alumnos en la mayoría de los grupos de trabajo mejoró en la segunda etapa al tener que subsanar las 
deficiencias observadas. Evaluar de forma crítica el trabajo de otros técnicos (en este caso compañeros de clase) forma parte de la competencia transversal responsabilidad profesional y ética. Aquellos alumnos que no fueron capaces de valorar de forma crítica la wiki creada de forma colaborativa obtuvieron una menor calificación.

Es importante llamar la atención sobre la "usabilidad" de las herramientas de PoliformaT. En este caso la edición de la wiki es un tanto rígida. Los alumnos se desmotivan cuando encuentran dificultades en la creación y edición de documentos. Por ello se plantea la posibilidad de en próximos cursos utilizar herramientas externas a la plataforma de la UPV pero de uso habitual entre los alumnos, tales como las que ofrece google drive. Se pretende con ello centrar la atención de los alumnos en la propia actividad y no tanto en la herramienta.

En el uso del foro los resultados observados fueron variados. A continuación, se muestra en las siguientes figuras los datos estadísticos ofrecidos por la herramienta estadísticas de PoliformaT sobre participación en la actividad.
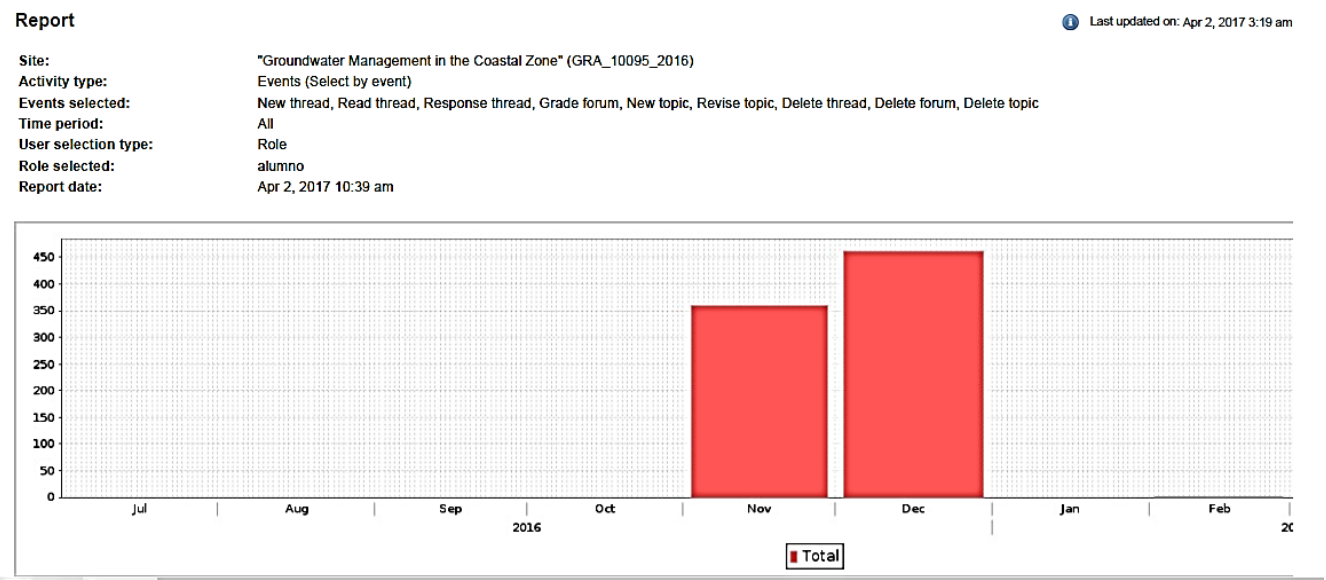

Fig. 3 Estadísticas de uso del foro en Groundwater management, rol alumno 


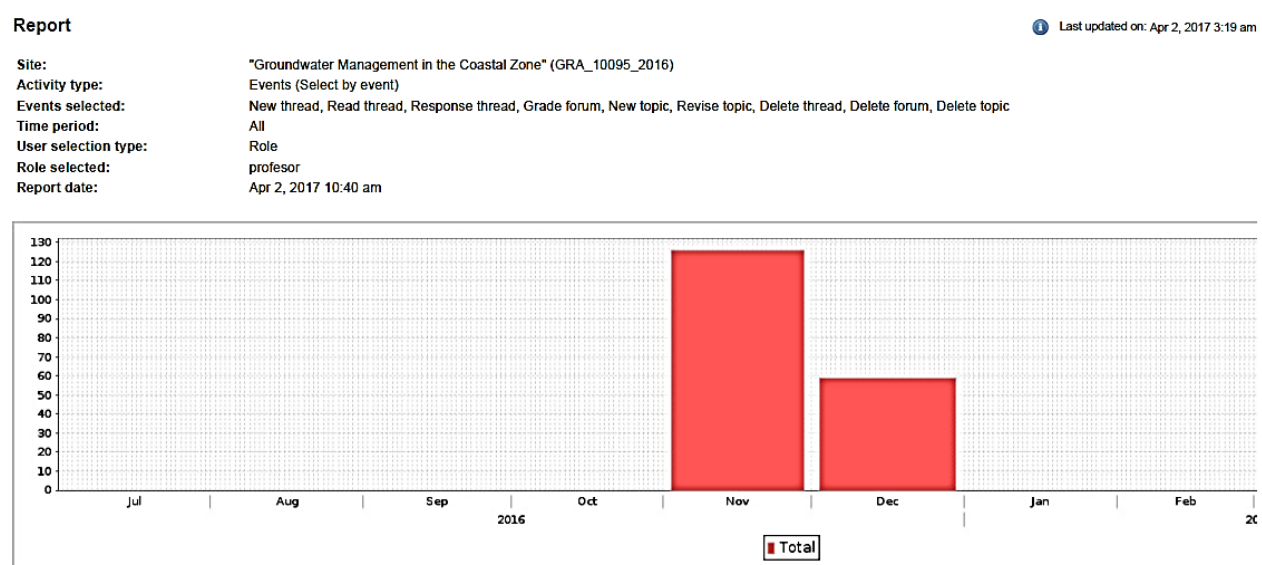

Fig. 4 Estadísticas de uso del foro en Groundwater management, rol profesor

La actividad en el foro empezó el día 29 de noviembre de 2016, por ello se muestra actividad en los meses de noviembre y diciembre. Las estadísticas muestran un total de 823 eventos por parte de los alumnos y 185 por parte del profesor. Se consideran como evento la siguientes acciones: Crear Nuevo hilo, Leer hilo, Responder al hilo, Grado foro, Crear Nuevo tema, Revisar tema, Eliminar hilo, Eliminar foro, Eliminar tema. De todos estos eventos los alumnos únicamente leyeron hilos de conversación o respondieron al hilo.

En la siguiente figura se muestra desglosada la actividad de los alumnos para las acciones responder y leer, obtenida desde la herramienta foro con su opción de generar estadísticas. La primera fila corresponde a la actividad de la profesora. Se ha eliminado el nombre de los alumnos por privacidad. 


\begin{tabular}{|c|c|c|c|}
\hline Authored $\bar{Z}$ & Read & Unread & Percent Read \\
\hline 32 & 119 & 0 & $100 \%$ \\
\hline 12 & 119 & 0 & $100 \%$ \\
\hline 11 & 97 & 22 & $82 \%$ \\
\hline 8 & 20 & 99 & $17 \%$ \\
\hline 8 & 15 & 104 & $13 \%$ \\
\hline 6 & 78 & 41 & $66 \%$ \\
\hline 5 & 10 & 109 & $8 \%$ \\
\hline 5 & 9 & 110 & $8 \%$ \\
\hline 5 & 10 & 109 & $8 \%$ \\
\hline 5 & 14 & 105 & $12 \%$ \\
\hline 5 & 59 & 60 & $50 \%$ \\
\hline 4 & 14 & 105 & $12 \%$ \\
\hline 4 & 41 & 78 & $34 \%$ \\
\hline 3 & 116 & 3 & $97 \%$ \\
\hline 3 & 40 & 79 & $34 \%$ \\
\hline 2 & 4 & 115 & $3 \%$ \\
\hline 1 & 2 & 117 & $2 \%$ \\
\hline 0 & 0 & 119 & $0 \%$ \\
\hline 0 & 1 & 118 & $1 \%$ \\
\hline 0 & 0 & 119 & $0 \%$ \\
\hline 0 & 1 & 118 & $1 \%$ \\
\hline
\end{tabular}

Fig. 5 Estadísticas del foro para las acciones responder (authored) y leer (read/unread)

En primer lugar, destacar que las estadísticas de lectura no son representativas. Para que se registre en el sistema la lectura de un comentario, el alumno debe seleccionar activamente la opción marcar como leído, y esto muchas veces no se hace. Así pues, la información más valiosa es el número y la calidad de sus contribuciones. Evaluando la contribución de cada alumno se puede conocer si el alumno ha leído en efecto los comentarios de sus compañeros, ya que se pueden detectar repeticiones, contradicciones, etc. A medida que transcurre el tiempo desde la apertura de la actividad es más complejo ser capaz de aportar nueva información relevante, por lo que los alumnos que realizan diversas aportaciones a lo largo de la actividad son aquellos con mejor nota. Ser capaz de matizar, criticar y mejorar lo aportado hasta el momento demuestra una mejor adquisición de la competencia. Destacar el rol de la profesora con una actividad moderadora continuada. La profesora no realiza en el foro una corrección directa de ninguno de los comentarios pero sí plantea dudas sobre estos que deben ser respondidas por los alumnos. Esta labor de moderación requiere un esfuerzo bastante relevante, y podría mejorarse con una ampliación de las posibilidades del foro. Por ejemplo, poder seguir las respuestas de un alumno dentro de cada conversación de forma automatizada permitiría una moderación y evaluación más eficiente. En la actualidad para seguir a un alumno es necesario releer uno a uno cada comentario.

(cc) EY-NC-ND 2017, Universitat Politècnica de València 


\section{Conclusiones}

El uso de herramientas de trabajo colaborativo es adecuado para trabajar y evaluar la competencia transversal Responsabilidad ética, medioambiental y profesional. En este sentido las herramientas ofrecidas por la plataforma de gestión del aprendizaje de la UPV, PoliformaT, son útiles pero susceptibles de alguna mejora. En concreto, la accesibilidad y alta de alumnos en el blog ha presentado dificultades técnicas que han empobrecido la participación del alumnado. En cuanto a la wiki, a los alumnos se les hace más difícil editar los contenidos que con otros tipos de herramientas a las que están más acostumbrados como las aplicaciones que ofrece google drive. Además, el resultado final que alcanzan con la wiki, en cuanto a formato es de menor calidad. Por último, las estadísticas y opciones automáticas del foro podrían ser ampliadas. En concreto, un seguimiento personalizado automático de las respuestas de cada alumno permitiría un moderación y evaluación más eficientes.

\section{Referencias}

ASENSIO-CUESTA, S.; GASCH, I.; PASCUAL-SEVA, N.; RUBIO, C.; VARGAS, M.; SEBASTIÁ-FRASQUET, M.T. (en prensa). "Poliblog como medio de presentación y evaluación de un proyecto de diseño" En Comunica2, Congreso Internacional sobre Redes Sociales ( $7^{\mathrm{a}}$ edición). Gandia. UPV.

FERNÁNDEZ MARCH, A., GIMÉNEZ CARBÓ, E., GÓMEZ NAVARRO, T., LOZANO AGUILAR, JOSÉ F., LÓPEZ SIEBEN, M., PASCUAL SEVA, N., SEGUÍ MÁS, E., TORMO CARBÓ, G., VARGAS COLÁS, M.A. (2017) "Rúbrica UPV CT-07. Responsabilidad ética, medioambiental y profesional".

GONZÁLEZ, J. WAGENAAR, R. (2003) “Tuning Educational Structures in Europe. Informe Final”. Bilbao: Universidad de Deusto.

PASCUAL-SEVA, N.; ASENSIO CUESTA, S.; SEBASTIÁ-FRASQUET, M.T.; VARGAS, M. (2015). "Evaluación de la competencia transversal UPV:"Responsabilidad ética, medioambiental y profesional" mediante herramientas de ludificación". En XXIII Congreso Universitario de Innovación Educativa en las Enseñanzas Técnicas (CUIEET 2015). Valencia: Escuela Técnica Superior de Ingeniería del Diseño. p. 250-259.

SEBASTIÁ-FRASQUET, M.T.; VARGAS, M.; ASENSIO CUESTA, S.; PASCUAL-SEVA, N. (2016). "Comparison of gamification tools for evaluating the ethical, environmental and professional responsibility skills in science degrees." En 9th International Conference of Education, Research and Innovation (ICERI 2016). Sevilla. IATED Academy. P 3609-3614.

UPV. Competencias Transversales. Available at: https://www.upv.es/entidades/ICE/info/U0724624.pdf. Last access date: 25/04/2017 